\title{
Subsurface excitations in a metal
}

Ray, M. P.; Lake, R. E.; Sosolik, C. E.; Thomsen, Lasse Bjørchmar; Nielsen, Gunver; Chorkendorff, Ib; Hansen, Ole

Published in:

Physical Review B Condensed Matter

Link to article, DOI:

10.1103/PhysRevB.80.161405

Publication date:

2009

Document Version

Publisher's PDF, also known as Version of record

Link back to DTU Orbit

Citation (APA):

Ray, M. P., Lake, R. E., Sosolik, C. E., Thomsen, L. B., Nielsen, G., Chorkendorff, I., \& Hansen, O. (2009). Subsurface excitations in a metal. Physical Review B Condensed Matter, 80(16), 161405.

https://doi.org/10.1103/PhysRevB.80.161405

\section{General rights}

Copyright and moral rights for the publications made accessible in the public portal are retained by the authors and/or other copyright owners and it is a condition of accessing publications that users recognise and abide by the legal requirements associated with these rights.

- Users may download and print one copy of any publication from the public portal for the purpose of private study or research.

- You may not further distribute the material or use it for any profit-making activity or commercial gain

- You may freely distribute the URL identifying the publication in the public portal 


\title{
Subsurface excitations in a metal
}

\author{
M. P. Ray, R. E. Lake, and C. E. Sosolik* \\ Department of Physics and Astronomy, Clemson University, Clemson, South Carolina 29634, USA \\ L. B. Thomsen, G. Nielsen, and I. Chorkendorff \\ Center for Individual Nanoparticle Functionality (CINF), Department of Physics, NanoDTU, Technical University of Denmark, \\ DK-2800 Kongens Lyngby, Denmark \\ O. Hansen \\ Center for Individual Nanoparticle Functionality (CINF), Department of Physics, NanoDTU, Technical University of Denmark, \\ DK-2800 Kongens Lyngby, Denmark and Department of Micro- and Nanotechnology, Technical University of Denmark (DTU), \\ Nanotech Building 345 East, DK-2800 Kgs. Lyngby, Denmark
}

(Received 23 July 2009; published 14 October 2009)

\begin{abstract}
We investigate internal hot carrier excitations in a Au thin film bombarded by hyperthermal and low energy alkali and noble gas ions. Excitations within the thin film of a metal-oxide-semiconductor device are measured revealing that ions whose velocities fall below the classical threshold given by the free-electron model of a metal still excite hot carriers. Excellent agreement between these results and a nonadiabatic model that accounts for the time-varying ion-surface interaction indicates that the measured excitations are due to semilocalized electrons near the metal surface.
\end{abstract}

DOI: 10.1103/PhysRevB.80.161405

PACS number(s): 79.20.Rf, 34.50.Bw, 34.50.Fa, 73.40.Qv

The development of a quantitative understanding of the mechanisms by which energy is dissipated in an ion-solid interaction is a fundamental problem ${ }^{1}$ whose solution directly impacts the fields of medicine, sensing technology, and materials modification. ${ }^{2-4}$ In the early 1960 s Lindhard and Scharff developed a theory that described the slowing of low energy heavy ions in a solid due to electronic excitations. ${ }^{5,6}$ To use this theory practically, however, stopping parameters had to be extracted from empirical fits to experimental data. Nevertheless, in this energy regime the theoretical results showed that stopping was proportional to the ion's velocity. 5,7 This is equivalent to Stokes' law of a velocitydependent stopping force that is commonly encountered in problems of viscous drag.

In 1989 Falcone and Sroubek developed a theory to describe electronic excitations due to energy losses of slow ions in solids which did not require empirically determined parameters and maintained a velocity-dependent stopping force. $^{8}$ Experimentally verifying the predictions of this theory have been problematic due to the difficulty of producing beams of slow ions and detecting their resulting excitations upon impact with a solid. Therefore, the few experimental studies performed in this energy regime have been limited to measurements outside a target where emitted excitations (electrons) can be collected. ${ }^{9,10}$

In particular, the work of Lakits et al. used the detection of externally emitted electrons to provide precise statistical information on the yield of electrons emitted per incident ion. ${ }^{9}$ Those data showed clear differences in the dependence of the yield on ion velocity, and distinct regimes for the yield were found that could be characterized by either potential emission (PE) or kinetic emission (KE). For example, in the limit of low velocities, species with ionization energies significantly greater than the surface work function gave a saturated PE yield that corresponded well with values specified by the depth of the ion's ionization level. With this PE satu- ration, low velocity kinetically induced electronic excitations were not resolved. With the introduction of ultrathin film solid-state devices into this field we can now detect kinetically induced electronic excitations within a solid for projectiles with low kinetic energies or velocities. This technique is similar to the first demonstrations of device-based detection of internal electronic excitations which were the adsorptiondriven creation of electron-hole pairs that arise in Schottky diode structures. ${ }^{11,12}$

In this Rapid Communication, we use ultrathin film metal-oxide-semiconductor (MOS) devices composed of $\mathrm{Au} / \mathrm{SiO}_{2} / \mathrm{n}-\mathrm{Si}(100)$ to measure hot carriers excited by beams of $\mathrm{He}^{+}, \mathrm{Li}^{+}, \mathrm{Ar}^{+}$, and $\mathrm{K}^{+}$ranging in energy from $100 \mathrm{eV}$ to 2 $\mathrm{keV}$. With the MOS device as our target, we probe both the electron and the hole excitations induced by the impinging beam. Our data confirm the predictions of the FalconeSroubek theory and reveal an unexpected interplay between lattice distortions and hole excitations that would be inaccessible using the external electron emission method.

The instrument used to conduct these measurements is a UHV low and hyperthermal energy Colutron G-2 ion gun and a custom built sample stage. ${ }^{13}$ The ion gun is capable of producing mass-resolved monoenergetic beams of noble gas and alkali ions. The beam exposure targets are large area ultrathin film MOS devices ${ }^{14}$ shown schematically in Fig. 1. The exposed surface of these devices consists of a $10 \mathrm{~nm} \mathrm{Au}$ layer on top of a $1 \mathrm{~nm}$ Ti wetting layer. The metal layers are separated from an $n$-doped $\mathrm{Si}(100)$ substrate by $5 \mathrm{~nm}$ of thermally grown silicon dioxide. Each device was electrically connected to the sample stage and shielded to limit the beam exposure to a small portion $8 \mathrm{~mm}^{2}$ of the $1 \mathrm{~cm}^{2} \mathrm{Au}$ top layer. All measurements were made with the ion beam focused at normal incidence to the device surface, and it was verified that photons emanating from the ion source created no measurable signal.

Multiple devices were exposed to beams of $\mathrm{He}^{+}, \mathrm{Li}^{+}, \mathrm{Ar}^{+}$, 

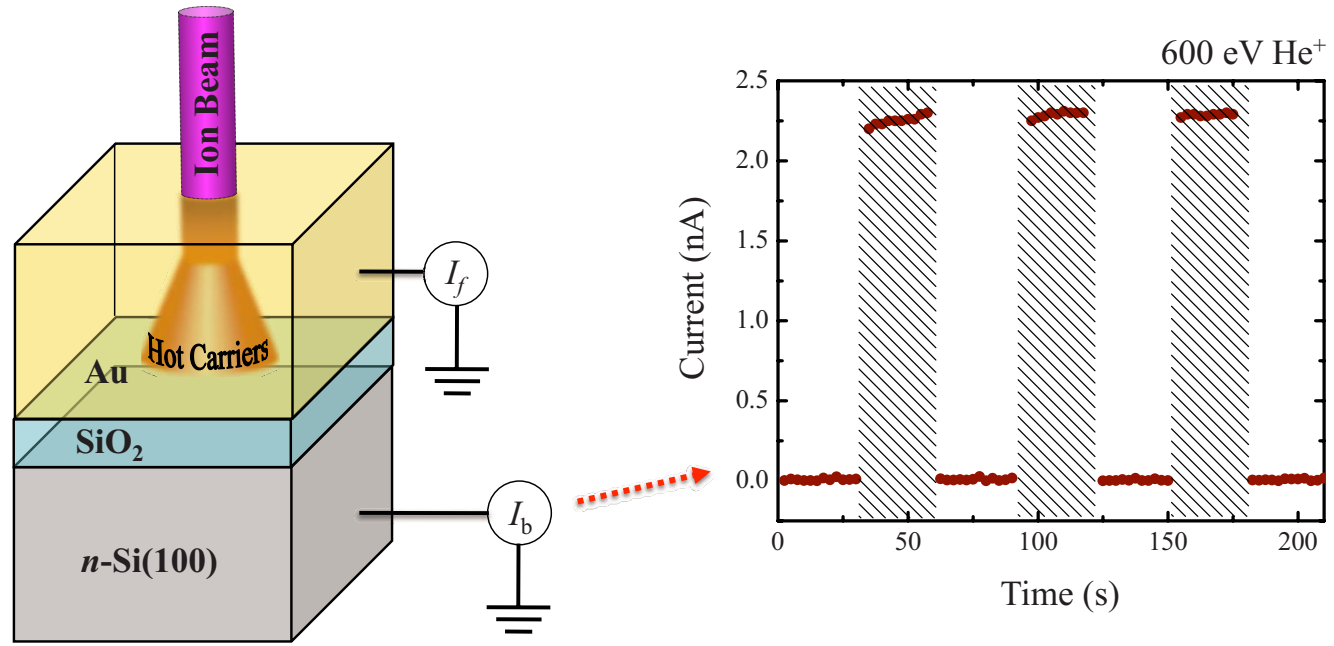

FIG. 1. (Color online) Cross section of MOS device under ion bombardment. The plot shows the hot-electron current measured through the MOS device in response to three periods of bombardment by $600 \mathrm{eV} \mathrm{He}$. The currents $I_{\mathrm{f}}$ and $I_{\mathrm{b}}$ were used to determine the kinetically induced hot-electron yield $\Gamma_{\mathrm{ie}}=\left|I_{b} /\left(I_{f}-I_{b}\right)\right|$. Shaded regions represent $30 \mathrm{~s}$ intervals when the device was exposed to the beam.

and $\mathrm{K}^{+}$, and the charge excitations observed for each species were consistent across the range of devices used. Excitations induced by the incident ion beam were measured at both frontside $\left(I_{\mathrm{f}}\right)$ metal and backside $\left(I_{\mathrm{b}}\right)$ Ohmic contacts of each exposed device as illustrated in Fig. 1, where a typical current response under a $\mathrm{He}^{+}$beam exposure is also shown. The I-V characteristics of each device, measured both pre- and postexposure, were compared to known results to verify that the device had not been altered due to beam effects. The internal hot carrier current was measured through the backside contact for each species as a function of the incidentbeam energy. We express this current response in terms of an internal excitation yield, $\Gamma_{\mathrm{ie}}$, that was used to quantify the kinetic energy or velocity-dependent effects for each device. Here $\Gamma_{\mathrm{ie}}=\left|I_{b} /\left(I_{f}-I_{b}\right)\right|$ is defined as the ratio of the magnitude of the backside current to the difference in the frontside and backside current responses (Fig. 1). The incident ion current and all charge carriers leaving the MOS top layer are registered by $I_{\mathrm{f}}$. Essentially, this top layer functions as a Faraday cup, and $I_{\mathrm{b}}$ registers the hot electronic excitations that can energetically "leak" from the device through the buried oxide interface. Our expression for $\Gamma_{\text {ie }}$ accounts for this energetic transport of hot charge carriers while also normalizing our yield for the incident-beam flux. This measurement scheme and representation of the yield are sensitive to the low energy subsurface kinetic excitations that are generated within the metal layer. Prior external measurements of electron emission in this velocity regime ${ }^{9}$ indicate a saturated or constant PE signal which should be present within our measured $I_{\mathrm{f}}$ values. However, as our measurement was not designed to collect or quantify these external excitations, we make no correction for them in our calculations of $\Gamma_{\text {ie }}$ and note that a constant $\mathrm{PE}$ contribution would represent a rigid shift in the yield and would not alter the physically relevant KE trend that is observed and discussed below.

Results for $\Gamma_{\mathrm{ie}}$ are shown in Fig. 2 for the four beam species used as a function of the incident-beam energy and beam velocity. It is clear that there is a near-zero response observed for $\mathrm{Ar}^{+}$and $\mathrm{K}^{+}$beams, while $\Gamma_{\text {ie }}$ values up to about
0.06 are observed for $\mathrm{He}^{+}$and $\mathrm{Li}^{+}$. In addition, the lighter mass species, $\mathrm{He}^{+}$, returns higher $\Gamma_{\text {ie }}$ values relative to $\mathrm{Li}^{+}$at a given incident energy. This inverse mass dependence is indicative of velocity-dependent excitations for $\mathrm{He}^{+}$and $\mathrm{Li}^{+}$, and as Fig. 2 shows, the data sets collapse to give a single velocity-dependent trend.

Velocity-dependent emission of electrons has historically been treated within the context of simple models that account for kinetic electron excitations (KEEs) by treating the target, in this case our top metal layer, as an idealized Fermi gas. Within such a model, a velocity threshold for kinetic electron emission, dependent only on the Fermi energy and the surface work function, can be found. ${ }^{15,16}$ For our work, we substitute the internal barrier height of the MOS device for the surface work function and obtain the following threshold velocity expression

$$
v_{t h}=\frac{1}{2} v_{f}\left[\left(1+\frac{\phi_{b}}{\mathrm{E}_{f}}\right)^{1 / 2}-1\right],
$$

where $\phi_{b}$ is the barrier height and $v_{f}$ and $\mathrm{E}_{f}$ are the Fermi velocity and Fermi energy for Au, respectively. The values for $\phi_{b}$ and $\mathrm{E}_{f}$ were chosen to be 4.2 and $5.5 \mathrm{eV}$. The dashed lines shown in Fig. 2 represent the threshold energy and velocity values obtained for $\mathrm{He}^{+}$and $\mathrm{Li}^{+}$, and it is clear that our experimentally observed excitations occur below this threshold. These data represent measurements of energydependent subthreshold excitations induced and transmitted internally in a solid, and as we show below, the detailed internal responses seen for $\mathrm{He}^{+}$and $\mathrm{Li}^{+}$allow us to isolate their origins in a way that would not be feasible in an external measurement.

Previous studies on subthreshold behavior in ion-solid interactions have attributed measured responses to four distinct mechanisms: electron promotion, multielectron processes, Auger excitations, and nonadiabatic interactions between the ion and the surface..$^{9,10,17-20}$ For our measurements we can rule out the first two mechanisms as the incident energies employed are too low for close-collision-induced promotion 

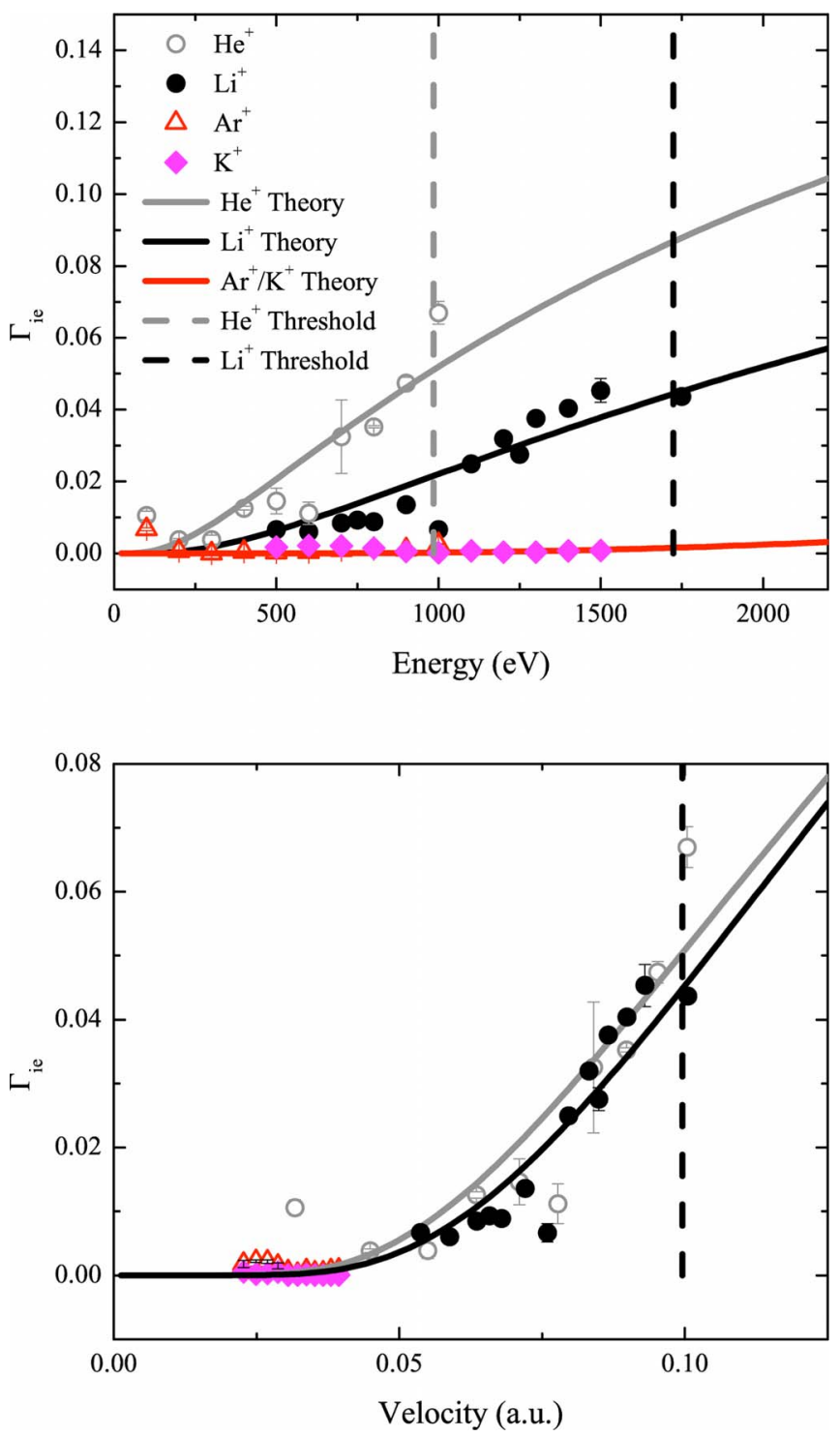

FIG. 2. (Color online) The upper panel shows current yield as a function of incident ion energy for four different ion species. The lower panel shows the same data plotted as a function of incident velocity. The solid lines are theoretical results from a model that describes charge-carrier excitations caused by ion-surface interactions. The vertical dashed lines represent the threshold for chargecarrier excitations using a free-electron model of a metal below which there should be no excitations.

and the projectiles are too simple (low Z) for multielectron effects to arise. Additionally, no Auger transitions analogous to those seen in previous systems exist for our projectiletarget combinations. Therefore, we are left to consider the role that nonabiabatic interactions play in creating our subthreshold signal responses. However, given the penetrating nature of the ions used here, it is also reasonable to suspect that depth-dependent effects could be present, especially for our thin Au films. To address this issue and determine the range of the ion species into the metal layer, we have used the simulations TRIM and SRIM. ${ }^{21}$ The ranges obtained for $\mathrm{He}^{+}$and $\mathrm{Li}^{+}$indicate that the mean depth obtained in both cases is less than $5.0 \mathrm{~nm}$ or half the thickness of the $\mathrm{Au}$ thin film. For the heavier species, the mean penetration depth is less than $1.2 \mathrm{~nm}$. As these values show, the various ion species do not penetrate the device beyond the top layer. That result, together with the clear velocity dependence seen in the yield data (Fig. 2), indicate that we can focus our analysis on nonadiabatic interactions with respect to the ion energy loss and the subsequent electronic excitations within the thin film.

Prior measurements of subthreshold electron emission external to a solid target have utilized the Falcone-Sroubek theory which is an extension of the concept of linear stopping power that is based on nonadiabatic interactions between the projectile ion and the metal atoms. ${ }^{10,18,22-24}$ Nonadiabaticity in this model is included via a time-varying ionsurface interaction that is characterized by the projectile velocity $v$, the potential $\mathrm{V}$, and a distance-dependent parameter $\gamma$ that accounts for the presence of the surface. ${ }^{25}$ Applying this model to our system, we can obtain a probablity of kinetic electron excitation $\mathrm{P}_{K E E}$ above the MOS barrier as

$$
P_{\text {KEE }}=\mathrm{A} \rho^{2} \mathrm{~V}^{2} \ln \left[\exp \left(-\frac{\pi \phi_{b}}{2 \gamma v}\right)+1\right]
$$

The parameters A and $\rho$ correspond to the collection efficiency of the MOS device and the target density of states, respectively. If we interpret our measurements of $\Gamma_{i e}$ as a direct measurement of this probability, we obtain the lines shown in Fig. 2 for each species. Here we have constrained $\gamma$ to be $\sim 1.0 \mathrm{au}$, which is its expected value within this model, and we obtain good agreement across the various incident species.

We can further determine the validity of this model by comparing the prefactor $\mathrm{A} \rho^{2} \mathrm{~V}^{2}$ obtained from our comparison of the data to Eq. (2) for $\mathrm{Li}^{+}$and $\mathrm{He}^{+}$to those obtained from SRIM. That is, within this model the electronic stopping is expressed as

$$
\frac{d E}{d x}=\frac{\pi}{3} \rho^{2} \mathrm{~V}^{2} k_{f}^{2} v
$$

where $k_{f}$ is the momentum of electrons on the Fermi sphere. ${ }^{8}$ The presence of the factor $\rho^{2} \mathrm{~V}^{2}$ in both Eqs. (2) and (3) imply that a ratio of prefactors from our data (Fig. 2) and SRIM are essentially equivalent ratios of species-specific factors. For our data we obtain a prefactor ratio $\left(\mathrm{Li}^{+} / \mathrm{He}^{+}\right)$of $0.8 \pm 0.1$. A linear fit to the stopping power as calculated within SRIM for these two species yields a value of 0.848 in excellent agreement with the experimental ratio.

The discussion above indicates that the response seen in our MOS devices under $\mathrm{He}^{+}$and $\mathrm{Li}^{+}$exposures can be interpreted quantitatively as an extension of linear stopping power into the subthreshold regime. Further analysis within TRIM shows that the penetration of the ions into our metal layer can lead to significant displacements of target atoms along a trajectory. This is most evident for $\mathrm{Li}^{+}$, where the electronic and nuclear stopping power are nearly equivalent across our incident energy range. Previous work has shown that when a lattice atom is displaced and the bandstructure is locally distorted, a narrow, transient subband is formed along the path of the penetrating ion. This is of particular relevance 


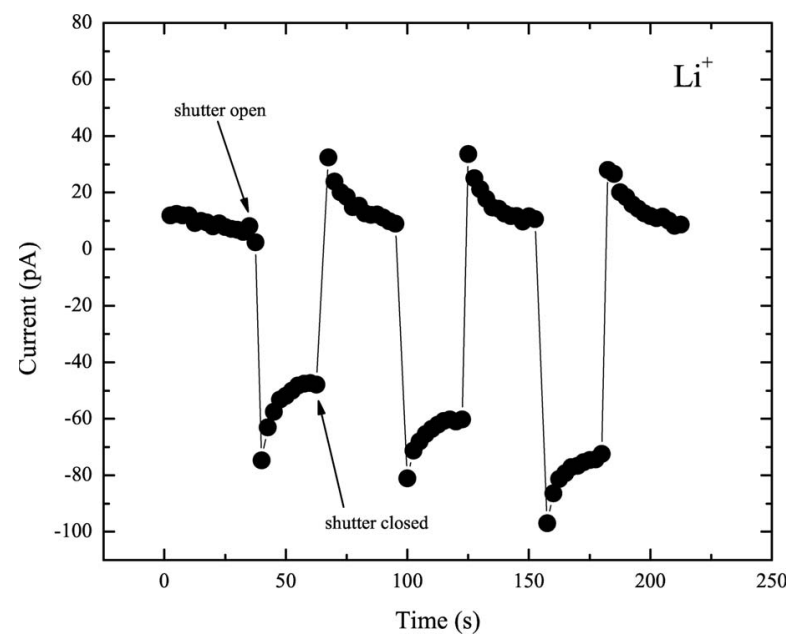

FIG. 3. Hot charge-carrier response during three periods of exposure for $1 \mathrm{keV} \mathrm{Li}^{+}$incident on an MOS device. The time-varying response is indicative of hot-hole interaction with oxide trap states.

because it has been shown that a transient subband can dramatically increase the mobility of hole excitations. ${ }^{26}$ In our $\mathrm{Li}^{+}$data, we observe a backside current response that is opposite in sign to that seen for all other species (Fig. 3). This is consistent with hole transport through our devices, and may indicate that target atom displacements induced by the ion alter the transport. Further evidence for hole tranport in the $\mathrm{Li}^{+}$case can be seen in Fig. 3, where the backside current response shows a unique time dependence that is analogous to that seen in chemicurrent measurements of nonreactive species on MOS devices. ${ }^{27}$ In that analysis, a sign-reversed and time-dependent response was attributed to interactions between hot holes and interface traps in the oxide layer of the MOS device.

In conclusion we have measured internal hot carrier excitations generated by the bombardment of MOS devices with hyperthermal and low energy alkali and noble gas ions. These excitations occur for projectile ion velocities that are below the threshold one obtains from a free-electron (binary collision) model and bridge the gap between adsorptiondriven excitations and excitations due to atomic collision cascades. ${ }^{28}$ To interpret these subthreshold results we have employed a nonadiabatic formalism that accounts for the time-varying potential between the ion and the target device. We find excellent agreement with our measured yields over the full range of energies studied. A unique aspect of these device-based measurements is that they provide the ability to discern the underlying mechanisms for energy dissipation through the solid matrix. As a demonstration of this concept, we have found a time-dependent hot-hole response for incident $\mathrm{Li}^{+}$beams. We interpret this transient response as an interaction between interface traps in the oxide layer of the MOS device and the hot-hole current. Simulated bombardment of the top metal layer indicates that $\mathrm{Li}^{+}$beams displace target atoms significantly along trajectory paths that extend well into the film. We suggest a mechanism where these displacements create a subband in the metal film which allows for efficient hole transport.

We gratefully acknowledge financial support from the National Science Foundation under Grant No. NSF-CHE0548111 and COMSET at Clemson University. The Center for Individual Nanoparticle Functionality is supported by the Danish National Research Foundation.

*sosolik@clemson.edu

${ }^{1}$ P. Sigmund, Stopping of Heavy Ions (Springer-Verlag, New York, 2004).

${ }^{2}$ E. Chason, J. Appl. Phys. 81, 6513 (1997).

${ }^{3}$ W. T. Chu, B. A. Ludewigt, and T. R. Renner, Rev. Sci. Instrum. 64, 2055 (1993).

${ }^{4}$ J. R. Castro, G. T. Y. Chen, and E. A. Blakely, Radiat. Res. 104, S263 (1985).

${ }^{5}$ J. Lindhard and M. Scharff, Phys. Rev. 124, 128 (1961).

${ }^{6}$ J. Lindhard, M. Scharff, and H. E. Schiøtt, Mat. Fys. Medd. K. Dan. Vidensk. Selsk. 33, 14 (1963).

${ }^{7}$ O. B. Firsov, Sov. Phys. JETP 9, 1076 (1959).

${ }^{8}$ G. Falcone and Z. Šroubek, Phys. Rev. B 39, 1999 (1989).

${ }^{9}$ G. Lakits, F. Aumayr, M. Heim, and H. Winter, Phys. Rev. A 42, 5780 (1990).

${ }^{10}$ J. Lörinčík, Z. Šroubek, H. Eder, F. Aumayr, and H. Winter, Phys. Rev. B 62, 16116 (2000).

${ }^{11}$ H. Nienhaus, H. S. Bergh, B. Gergen, A. Majumdar, W. H. Weinberg, and E. W. McFarland, Phys. Rev. Lett. 82, 446 (1999).

${ }^{12}$ B. Gergen, H. Nienhaus, W. H. Weinberg, and E. W. McFarland, Science 294, 2521 (2001).

${ }^{13}$ M. P. Ray, R. E. Lake, S. A. Moody, V. Magadala, and C. E. Sosolik, Rev. Sci. Instrum. 79, 076106 (2008).

${ }^{14}$ L. B. Thomsen, G. Nielsen, S. B. Vendelbo, M. Johansson, O. Hansen, and I. Chorkendorff, Phys. Rev. B 76, 155315 (2007).

${ }^{15}$ R. Baragiola, Nucl. Instrum. Methods Phys. Res. B 78, 223

(1993).

${ }^{16}$ R. Baragiola, E. Alonso, J. Ferron, and A. Oliva-Florio, Surf. Sci. 90, 240 (1979)

${ }^{17}$ R. A. Baragiola, E. V. Alonso, and A. Oliva Florio, Phys. Rev. B 19, 121 (1979).

${ }^{18}$ J. A. Yarmoff, T. D. Liu, S. R. Qiu, and Z. Šroubek, Phys. Rev. Lett. 80, 2469 (1998).

${ }^{19}$ Z. Šroubek, X. Chen, and J. A. Yarmoff, Phys. Rev. B 73, 045427 (2006).

${ }^{20}$ J. A. Yarmoff, H. T. Than, and Z. Šroubek, Phys. Rev. B 65, 205412 (2002).

${ }^{21}$ J. Biersack and L. Haggmark, Nucl. Instrum. Methods Phys. Res. 174, 257 (1980).

${ }^{22}$ P. Varga and H. P. Winter, Particle Induced Electron Emission II (Springer, Heidelberg, 1992), pp. 149-214.

${ }^{23}$ H. Eder, F. Aumayr, and H. P. Winter, Nucl. Instrum. Methods Phys. Res. B 154, 185 (1999).

${ }^{24}$ H. P. Winter, H. Eder, F. Aumayr, J. Lorincik, and Z. Sroubek, Nucl. Instrum. Methods Phys. Res. B 182, 15 (2001).

${ }^{25}$ J. Lörinčík and Z. Šroubek, Nucl. Instrum. Methods Phys. Res. B 164-165, 633 (2000).

${ }^{26}$ Z. Šroubek, Phys. Rev. Lett. 70, 4019 (1993).

${ }^{27}$ B. Roldan Cuenya, H. Nienhaus, and E. W. McFarland, Phys. Rev. B 70, 115322 (2004).

${ }^{28}$ S. Meyer, D. Diesing, and A. Wucher, Phys. Rev. Lett. 93, 137601 (2004). 